\title{
Depth dependence of residual strains in polycrystalline Mo thin films using high-resolution x-ray diffraction
}

\author{
S. G. Malhotra, Z. U. Rek, ${ }^{\text {a) }}$ S. M. Yalisove, and J. C. Bilello \\ Department of Materials Science and Engineering, University of Michigan, Ann Arbor, MI 48109-2136
}

(Received 31 July 1995; accepted for publication 20 January 1996)

\begin{abstract}
The magnitude of the stress in a thin film can be obtained by measuring the curvature of the film-substrate couple. Crystal curvature techniques yield the average stress throughout the film thickness. On a microscopic level, the details of the strain distribution, as a function of depth through the thickness of the film, can have important consequences in governing film quality and ultimate morphology. A new method, using high-resolution x-ray diffraction to determine the depth dependence of strain in polycrystalline thin films, is described. The technique requires an analysis of the diffraction peak shifts of at least six independent $\{h k l\}$ scattering vectors, at a variety of penetration depths from the free surface of the film. The data are then used to determine the magnitude and directions of the strain eigenvalues in a laboratory reference frame for each penetration depth from the free surface of the film. A linear elastic model was used to determine the strains in successive slabs of the film. Results are reported for two Mo films, with nominal thicknesses of 50 and $100 \mathrm{~nm}$, which were deposited by planar magnetron sputtering onto $\mathrm{Si}$ (100) substrates. This technique can provide quantitative insight into the depth variation of residual strains (stresses) in thin films and should work with a wide variety of materials. (C) 1996 American Institute of Physics. [S0021-8979(96)03209-6]
\end{abstract}

\section{INTRODUCTION}

The development of residual strains during the growth of polycrystalline thin films can compromise the integrity of the structure. For example, the presence of residual strains may have a significant effect on the final microstructure, e.g., the grain size and the degree and nature of the texture. ${ }^{1}$ Deviations in the expected microstructure could then cause undesirable modifications to the mechanical, optical, electrical, and/or magnetic properties of the film. ${ }^{2}$ In the worst case, the presence of excessive tensile strains can lead to film and substrate cracking, and excessive compressive strains can produce film decohesion by buckling.

There are two general classes of commonly used techniques to quantify the amount of strain (or stress) present in a thin film:

(1) deflection techniques based on determining the radius of curvature of the substrate; $;^{3-12}$ and

(2) strain measurement techniques based on the direct measurements of interplanar spacings in the film using $\mathrm{x}$-ray diffraction. ${ }^{13-22}$

The substrate deflection techniques include optical interferometry, ${ }^{4}$ laser scanning, ${ }^{5}$ and double-crystal diffraction topography (DCDT). ${ }^{9}$ Optical interferometry and laser scanning determine the radius of curvature of the physical surface of the substrate $R$ which equals the inverse of the curvature $K^{4,5}$ The DCDT technique, on the other hand, determines the curvature of the crystal lattice planes of the substrate near the film-substrate interface by measuring the distance between successive Bragg angle contours. ${ }^{6-8}$ The distance between the contours is then proportional to the

${ }^{a)}$ Stanford Synchrotron Radiation Laboratory, Stanford, CA 94309. curvature, $K .^{9,10}$ The resulting curvatures obtained from the above techniques are then linearly related to the average stress using standard equations. ${ }^{11,12}$

Other x-ray scattering methods have been used to determine the residual strains. These include the $\sin ^{2} \psi \mathrm{x}$-raydiffraction technique ${ }^{17-19}$ and $\mathrm{x}$-ray scattering in a grazing incidence geometry. ${ }^{20-22}$ As with the curvature techniques discussed above, the $\sin ^{2} \psi$ method can only determine the average stress because the scattered volume includes the entire film thickness. When conducting x-ray-scattering experiments in a grazing incidence geometry, however, one can use the phenomenon of total external reflection of the incident $\mathrm{x}$-ray beam to control depth of penetration. This depth sensitivity has been used to avoid noise from the substrate ${ }^{23}$ and to profile the strains in crystallographic planes perpendicular to the sample surface as a function of penetration depth. ${ }^{24-26}$ Finally, still other x-ray-diffraction methods have been developed to determine the average strain (or stress) tensor for thin films, ${ }^{27-30}$ but these cannot be readily extended to do depth profiling.

In contrast to this previous work, the goal of the present study was to use high-resolution $\mathrm{x}$-ray diffraction to determine the strain in crystallographic planes with a variety of orientations in three-dimensional space, as a function of $\mathrm{x}$-ray penetration depth, in nominally 50- and 100-nm-thick Mo films. The scattering geometries used are commonly referred to as symmetric and asymmetric grazing incidence geometries. ${ }^{25}$ The strain data were used to determine the magnitude and orientation of the principal strains, or strain eigenvalues and eigenvectors, respectively, at each depth of penetration. After the strain eigenvectors were resolved onto a laboratory reference frame for each depth, a linear elastic model was used to calculate the average strains in successive slabs of the film. Mo was used because it has a high atomic 
number suitable for scattering efficiency, and it has the potential for a variety of useful technical applications. ${ }^{31-32}$

\section{EXPERIMENTAL PROCEDURES}

\section{A. Sample preparation}

Molybdenum films with thicknesses of $50 \pm 2.5$ and $100 \pm 5 \mathrm{~nm}$ [as determined by Rutherford backscattering $(\mathrm{RBS})^{33}$ ] were deposited from a $99.95 \%$ pure Mo target onto 75-mm-diam Si (100) wafers by direct-current planar magnetron sputtering. The wafers were in the "as-received" condition, with a native oxide coating. The sputtering occurred without significant heating of the substrates. The sputtering power was $308 \mathrm{~W}(\sim 8 \mathrm{~nm} / \mathrm{min}$ deposition rate $)$ and the chamber was pumped to a base pressure of $\sim 6 \times 10^{-6}$ Torr. The chamber was then back-filled with Ar and maintained at a pressure of $10 \mathrm{mTorr}$. The samples were mounted face down $5 \mathrm{in}$. above the sputter source in a horizontal carousel which rotated at $20 \mathrm{rpm}$. The target was presputtered onto the shutter for at least $1 \mathrm{~min}$ to prevent oxides or contaminants from being sputtered on to the wafers.

\section{B. Measurement}

The present high-resolution diffraction experiments were conducted under standard synchrotron conditions $(3 \mathrm{GeV}$ and $100 \mathrm{~mA}$ at fill) on the eight-pole focused wiggler station BL 7-2 at the Stanford Synchrotron Radiation Laboratory (SSRL). A Si (111) double-crystal monochromator was used to select the incident $\mathrm{x}$-ray wavelength of $0.124 \mathrm{~nm}(10 \mathrm{keV})$ from the continuous spectrum. The horizontal and vertical divergence of the beam on BL 7-2 is 3 and $0.2 \mathrm{mrad}$, respectively. Slits $1 \times 1 \mathrm{~mm}^{2}$ were used for the incoming beam, and $1 \mathrm{mrad}$ Soller slits were used for the diffracted beam to limit vertical divergence. The signal was detected with a scintillation counter. The samples were mounted on an automated Huber 5020 four-circle goniometer. The dedicated beam-line 7-2 computer was used to control the goniometer motions, the shutter, and the photon counting. The experiments were conducted in the "dose" mode by putting a scintillation counter in the path of the incident beam, because the current in the synchrotron ring decreased linearly with time.

The symmetric and asymmetric grazing incidence geometrie $^{25}$ were used to collect the data so that crystallographic planes with a variety of orientations could be probed, and are illustrated in Fig. 1. The diffraction condition can be satisfied for crystallographic planes which are nearly perpendicular to the sample surface in the symmetric geometry, whereas the diffraction condition can be satisfied for planes which are inclined with respect to the sample surface in the asymmetric geometry. The diffraction peaks collected in the symmetric and asymmetric geometries, respectively, at each penetration depth for each film, are shown in Tables I and II. The penetration depths were varied by changing the angle of the incoming radiation near the critical angle for total external reflection, as shown in Fig. $2{ }^{34}$ The penetration depths were accurately determined to within $\pm 5 \mathrm{~nm}$, except very near the critical angle, where the resolution was $\pm 15 \mathrm{~nm}$. (a)

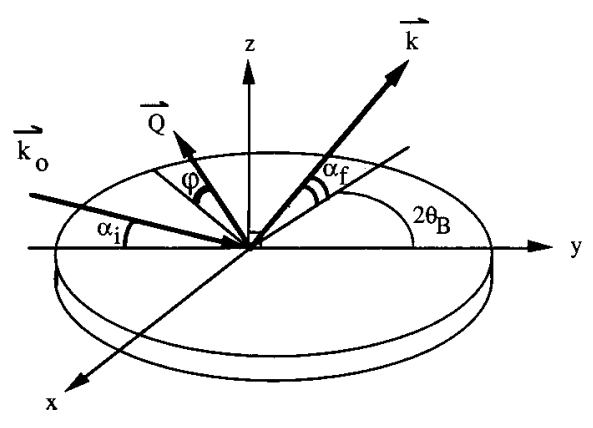

(b)

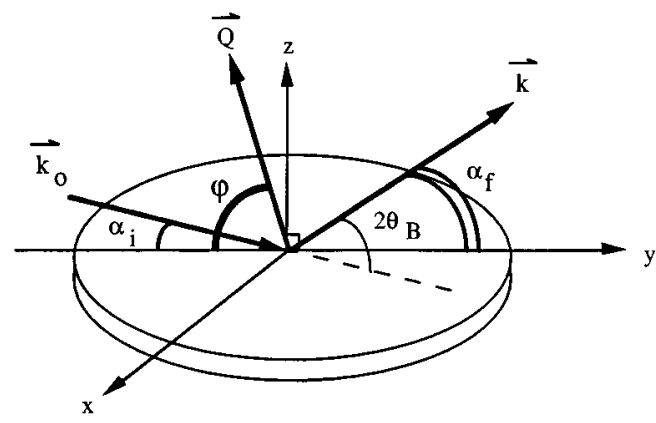

FIG. 1. Schematic of (a) symmetric grazing incidence geometry, and (b) asymmetric grazing incidence geometry. For the symmetric case, the incident beam $\mathbf{k}_{\mathbf{0}}$ makes a grazing angle $\alpha_{i}<1^{\circ}$ with respect to the sample surface and the diffracted beam $\mathbf{k}$ exits at an angle $\alpha_{f} \approx \alpha_{i}$. The $\mathbf{Q}$ vector is then nearly parallel to the sample surface with the angle $\varphi<1^{\circ}$. For the asymmetric case, the incident beam $\mathbf{k}_{\mathbf{0}}$ makes a grazing angle $\alpha_{i}<1^{\circ}$ with respect to the sample surface and the diffracted beam $\mathbf{k}$ exits at angle $\alpha_{f} \neq \alpha_{i}$. The $\mathbf{Q}$ vector is then inclined with respect to the sample surface. The angle $\varphi$ is $\gg 1^{\circ}$. As shown, the planes which can satisfy the Bragg condition in this case are inclined with respect to the sample surface.

\section{RESULTS}

The diffraction data were corrected for Lorentz polarization and absorption and fit with a Voigt or Gaussian function to determine the location of each peak, $2 \theta$. This was then used to determine the interplanar spacings for each $\{h k l\}$ family of planes $d_{h k l}$ from Bragg's law and the strain was calculated using $\epsilon_{h k l}=\left(d_{h k l}-d_{0}\right) / d_{0}$. Because of the difficulties involved in making a strain-free refractory metal film standard, a strain-free lanthanum hexaboride $\left(\mathrm{LaB}_{6}\right)$ powder standard (NIST Standard Reference Material 660) was used to detect any systematic offset during the experiment. The

TABLE I. List of diffraction peaks collected at each penetration depth from the free surface of the 50-nm-thick Mo film.

\begin{tabular}{ccc}
\hline \hline & \multicolumn{2}{c}{ Peaks collected } \\
\cline { 2 - 3 } $\begin{array}{c}\text { Penetration } \\
\text { depth }(\mathrm{nm})\end{array}$ & $\begin{array}{c}\text { Symmetric } \\
\text { geometry }\end{array}$ & $\begin{array}{c}\text { Asymmetric } \\
\text { geometry }\end{array}$ \\
\hline 10 & $\cdots$ & $\{110\},\{200\},\{211\}$ \\
& $\ldots 110\},\{200\}$, & $\{110\},\{200\},\{211\}$ \\
& $\{211\},\{310\}$ & \\
& $\{222\},\{321\},\{400\}$ & \\
\hline \hline
\end{tabular}


TABLE II. List of diffraction peaks collected at each penetration depth from the free surface of the 100-nm-thick Mo film.

\begin{tabular}{ccc}
\hline \hline & \multicolumn{2}{c}{ Peaks collected } \\
\cline { 2 - 3 } $\begin{array}{c}\text { Penetration } \\
\text { depth }(\mathrm{nm})\end{array}$ & $\begin{array}{c}\text { Symmetric } \\
\text { geometry }\end{array}$ & $\begin{array}{c}\text { Asymmetric } \\
\text { geometry }\end{array}$ \\
\hline 2.5 & $\{110\}$ & $\{110\},\{200\},\{211\}$ \\
5 and 10 & $\{110\},\{200\}$, & $\{110\},\{200\},\{211\}$ \\
& $\{211\},\{220\}$, & \\
& $\{310\},\{321\}$ & \\
& $\{110\},\{200\}$, & $\{110\},\{200\},\{211\}$ \\
$20,55,75$, and 100 & $\{211\},\{220\}$ & \\
& $\{310\},\{222\}$, & \\
& $\{321\},\{400\}$ & \\
\hline \hline
\end{tabular}

Joint Committee for Powder Diffraction Standards' (JCPDS) $d_{0}$ values for Mo were then corrected for this offset and used for the strain calculations. The resulting strains for the planes collected in the symmetric geometry, as a function of depth of penetration, for the 100-nm-thick film are shown in Fig. 3. This is representative of the strains for the 50-nm-thick film as well. The strains for the planes collected in the asymmetric geometry, as a function of depth of penetration, are shown for both films in Fig. 4.

The strain tensors were calculated for the penetration depths in each film for which at least six diffraction peaks were collected using the least-squares methodology developed by Imura and co-workers. ${ }^{27}$ For the $2.5 \mathrm{~nm}$ penetration depth, the collection times became impractical for the higher-order diffraction peaks. Thus, only four lower-order peaks were collected and the strain tensor was not determined. The precision of the least-squares approach is better than those which use only six independent strains to solve the strain tensor. The laboratory reference frame used for the tensor calculations is shown in Fig. 5. The $x, y$, and $z$ axes in the laboratory frame were defined by considering the orientation of the plane of diffraction in the asymmetric geometry. The diffraction plane contains the incoming $\mathrm{x}$ ray $\mathbf{k}_{\mathbf{0}}$, the diffracted $\mathrm{x}$ ray $\mathbf{k}$, and the sample normal $\mathbf{n}$. Thus, the sample normal defined the $z$ axis, the perpendicular compo-

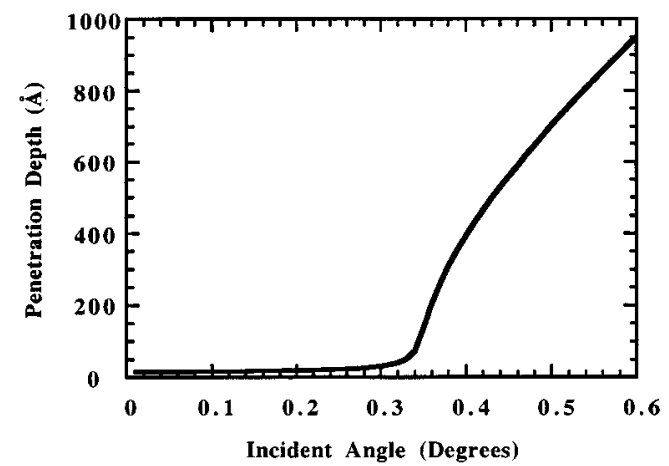

FIG. 2. Plot of $1 / e$ penetration depth for $0.124 \mathrm{~nm} \mathrm{x}$ rays into Mo for grazing incidence angles. Refraction leads to a severe reduction in the penetration depth when $\alpha_{i}$ is less than $0.34^{\circ}$, the critical angle for total external reflection for Mo. Above the critical angle the 1/e penetration depth approaches that for large angles.
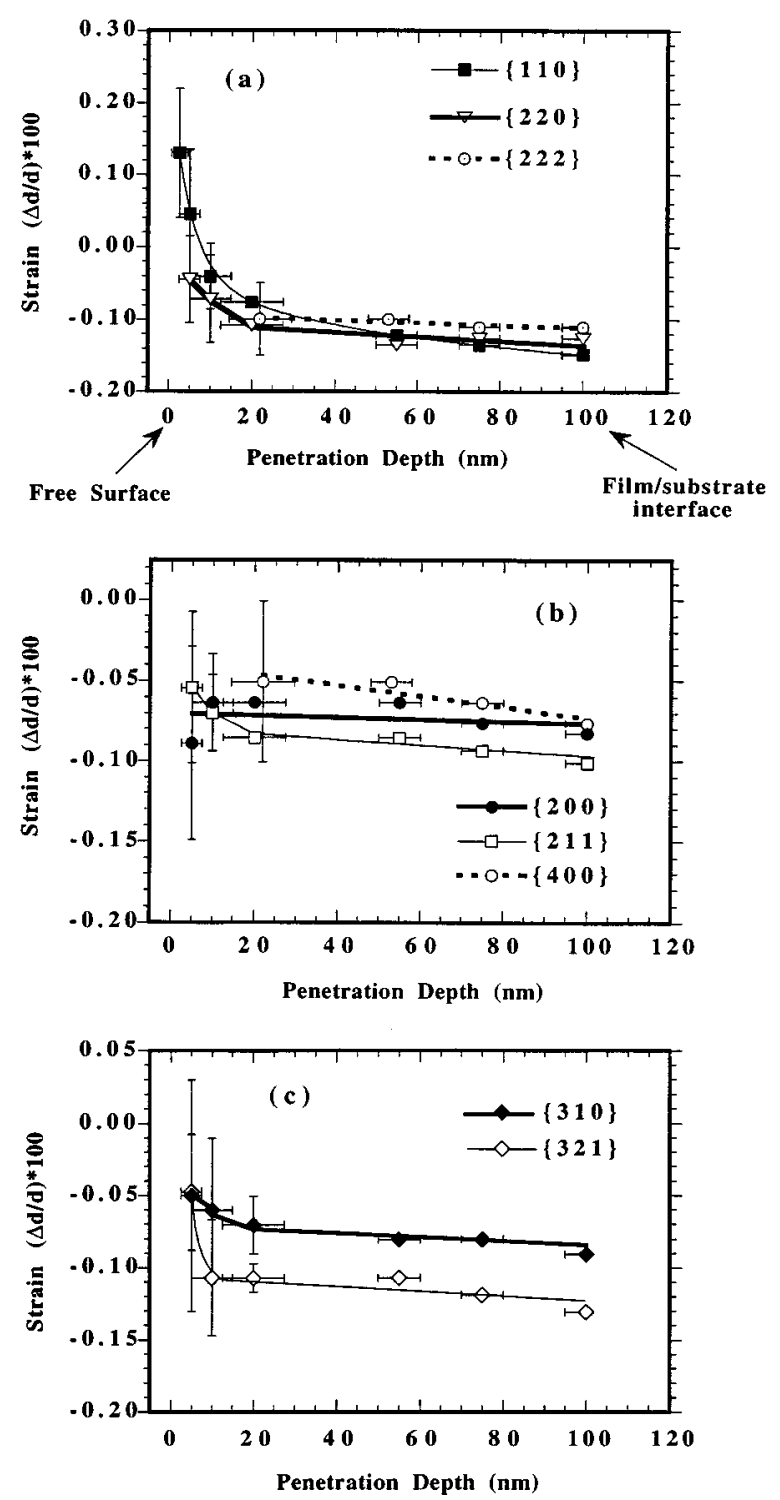

FIG. 3. Strains in planes perpendicular to the sample surface, obtained in symmetric grazing incidence geometry, for 100-nm-thick Mo film for (a) $\{110\},\{220\}$, and $\{222\}$ planes. (b) $\{200\},\{211\}$, and $\{400\}$ planes; and (c) $\{310\}$ and $\{321\}$ planes There is not a significant strain gradient through the thickness of the film.

nent to the plane of diffraction defined the $x$ axis, and the cross product of $x$ and $z$ defined the $y$ axis. The Si [110] direction is parallel to the $y$ axis, and this direction was placed perpendicular to the axis of rotation, and parallel to the tangent of the platen, during sputter deposition. Once defined in the laboratory reference frame, all strain displacement vectors were used to solve for the six unknowns in the strain tensor. The strain eigenvalues and eigenvectors were then calculated for each symmetric strain tensor using the approach outlined by Nye. ${ }^{35}$ The strain eigenvalues of a known orientation were resolved onto the laboratory axes defined in Fig. 5 and were converted to stresses using Hooke's law. ${ }^{36}$ The isotropic modulus was used in the Hooke's law calculation because Mo films less than $100 \mathrm{~nm}$ in thickness have been shown to be random and polycrystalline, with a small grain size on the order of $25 \mathrm{~nm}^{37,38}$ 

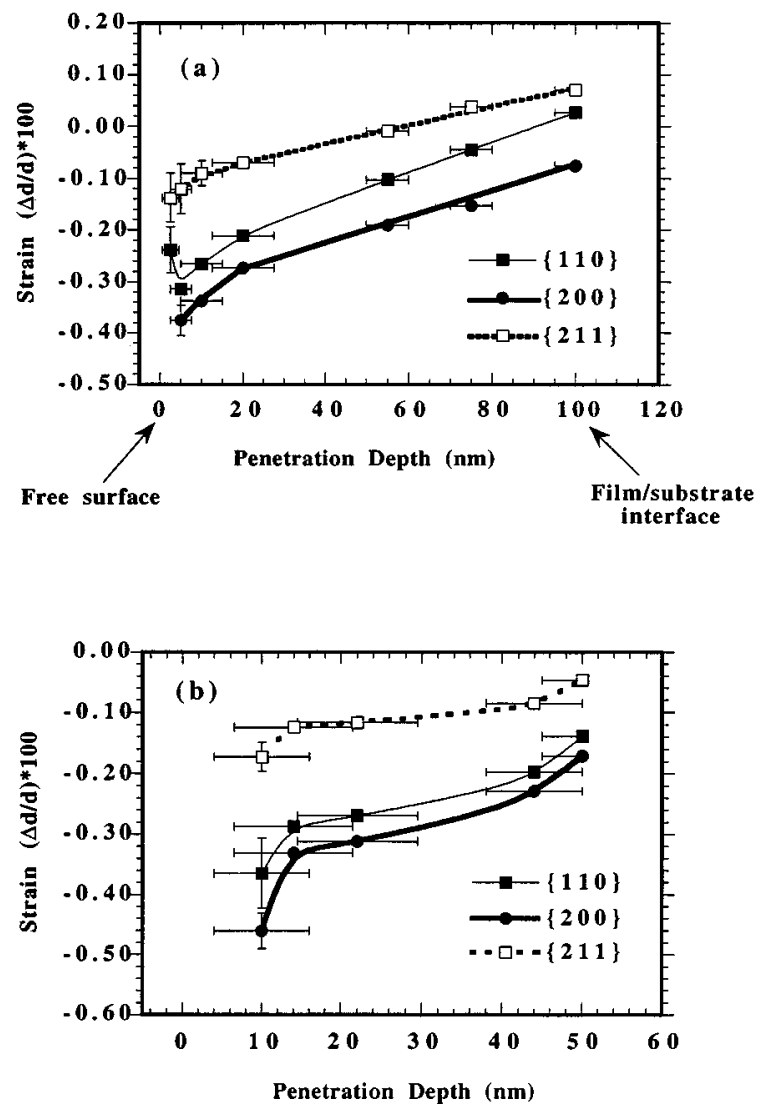

FIG. 4. Strains in planes inclined to the sample surface, obtained in asymmetric grazing incidence geometry, for (a) 100-nm-thick Mo film for $\{110\}$, $\{200\}$, and $\{211\}$ planes and (b) 50-nm-thick Mo film for $\{110\},\{200\}$, and $\{211\}$ planes. There is a strain gradient for each film, which decreases in magnitude as the penetration depth from the free surface increases.

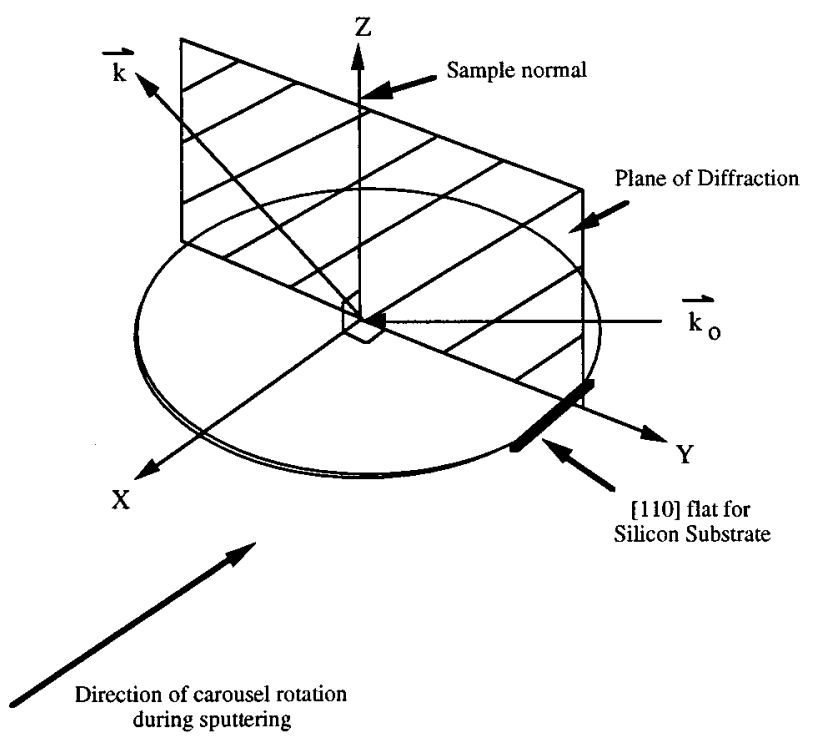

FIG. 5. Schematic of laboratory axis system used for strain eigenvalue and eigenvector calculations. The sample normal, incoming $\mathrm{x}$ ray, $\mathbf{k}_{\mathbf{0}}$, and outgoing $\mathrm{x}$ ray, $\mathbf{k}$, comprise the diffraction plane. The sample normal forms the $z$ axis, the perpendicular to the diffraction plane forms the $x$ axis, and the cross product of the $x$ and $z$ axes forms the $y$ axis. Note that the [110] flat for the silicon substrate is perpendicular to the $y$ axis.
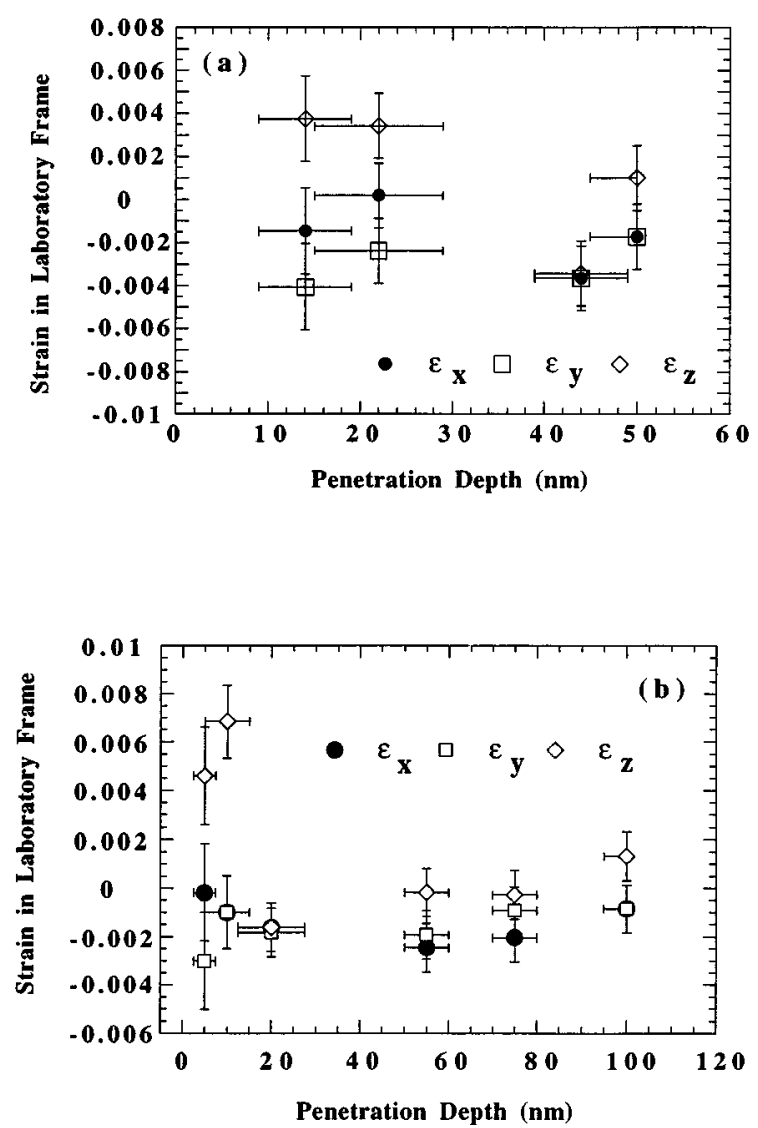

FIG. 6. Strain eigenvalues resolved onto the laboratory frame as a function of penetration depth for (a) 50-nm-thick film and (b) 100-nm-thick Mo film. A gradient in the normal strain $\epsilon_{z}$, is evident near the free surface of the film. The strain eigenvalues were not determined for the $2.5 \mathrm{~nm}$ penetration depth because it was not feasible to collect a sufficient number of diffraction peaks.

Therefore, the isotropic elastic approach for these films was a reasonable approximation. The strain eigenvalues resolved onto the laboratory frame as a function of penetration depth are shown in Fig. 6 for the 50- and 100-nm-thick films.

As previously mentioned, the strain eigenvectors resolved onto the laboratory reference frame as a function of penetration depth from the free surface, shown in Fig. 6, can be converted to the magnitudes of the strains in successive slabs of the film. This can be obtained from a linear elastic model, which was used because the conventional approach to determine the " $z$ profile" or "true gradient" of strain is not applicable to very thin films where the penetration depth can equal the total film thickness. ${ }^{39,40}$ Consider a volume of material for which the strain is known in the $x, y$, and $z$ directions for three penetration depths from the free surface. The volume can then be divided into slabs of thickness $t_{i}$, where $i=1,2$, or 3 , as illustrated in Fig. 7. Then the measured strain for a variety of penetration depths from the free surface $\epsilon_{J}^{N}$, is equal to a weighted summation of the strains present in each slab $\epsilon_{J}^{i}$ of thickness $t_{i}$,

$$
\epsilon_{J}^{N}=\frac{\sum_{i=1}^{N} \epsilon_{J}^{i} t_{i}}{\sum_{i=1}^{N} t_{i}}
$$




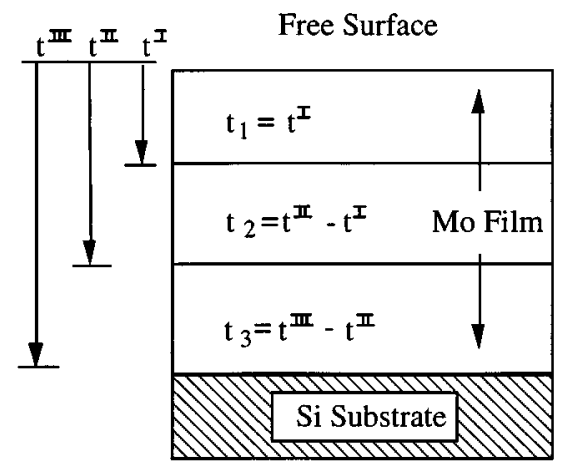

FIG. 7. Schematic illustrating method used to divide film into slabs of thickness $t$.

where $N$ is the number of penetration depths from the free surface, and $J$ is the $x, y$, or $z$ direction. Using these equations, the strains present in each slab can be calculated and can also be summed to obtain the strain in larger volumes

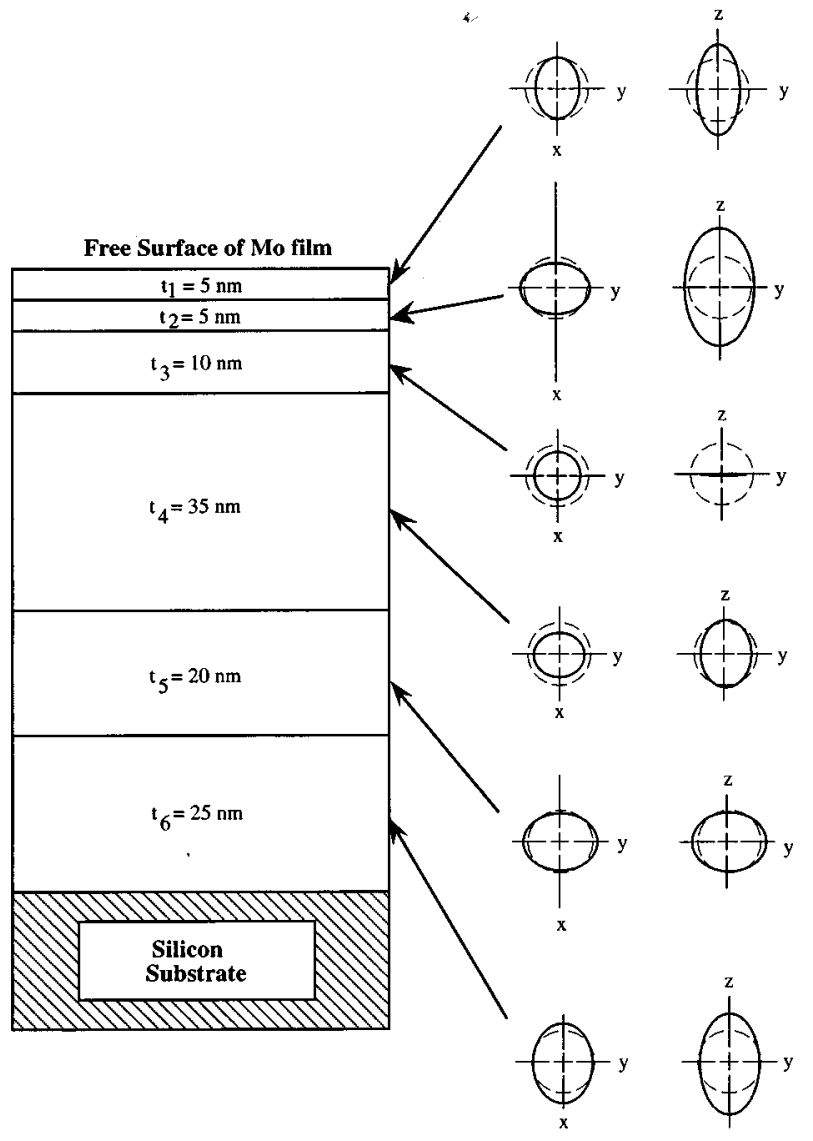

FIG. 8. Schematic illustrating strain ellipsoids in each slab in the 100-nmthick film. The solid ellipses represent the magnitude of the strains $\epsilon_{x}, \epsilon_{y}$, and $\epsilon_{z}$ in the laboratory frame and are drawn with respect to a dashed unit circle which represents a volume element in the unstrained body. The radius of the ellipse in a direction that was subjected to a tensile strain is greater than unity, and the radius in a direction that was subjected to a compressive strain is less than unity. The strains in the $x, y$, and $z$ directions were increased by 50 times so that the differential with the unit sphere is discernible. The strain ellipsoid was not determined for the $2.5 \mathrm{~nm}$ penetration depth because it was not feasible to collect a sufficient number of diffraction peaks.

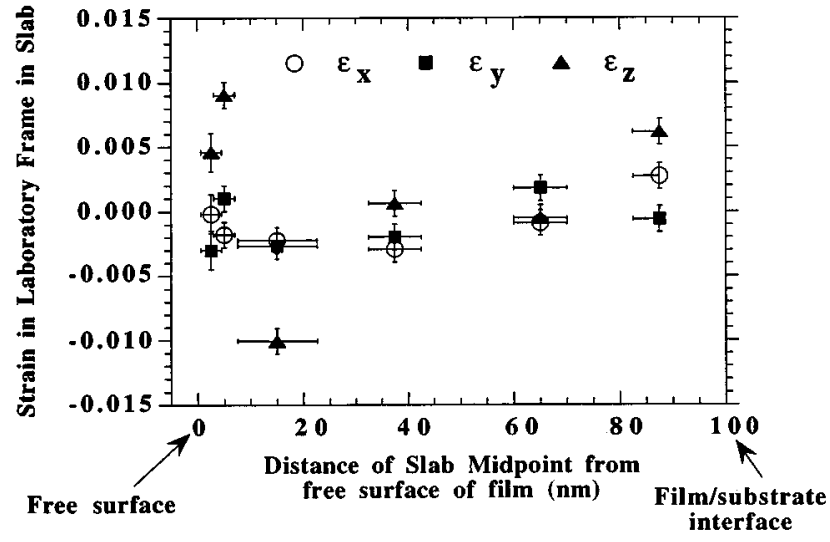

FIG. 9. Plot of strains in slabs of the $100 \mathrm{~nm}$ film which were depicted schematically as strain ellipsoids in Fig. 8.

originating from the film/substrate interface. A schematic which illustrates the strain ellipsoids determined for each slab in the 100-nm-thick film is shown in Fig. 8, and a plot of the strains in each slab is shown in Fig. 9.

\section{DISCUSSION}

The strains for planes perpendicular to the sample surface as a function of penetration depth shown in Fig. 3 for the 100-nm-thick film display a negligible gradient, and a surface relaxation appears for penetration depths less than 10 $\mathrm{nm}$. This is the same trend previously observed for strains in crystallographic planes perpendicular to the sample surface in $\mathrm{Al},{ }^{24,41}$ and $\mathrm{Al}-2 \% \mathrm{Cu},{ }^{26}$ films. Thus, a variety of thin films appear to be strained isotropically in the plane. However, a significant strain gradient was identified in crystallographic planes inclined to the sample surface for both films, as shown in Fig. 4, which would agree with investigations that have implied a strain gradient in Mo and other thin films. ${ }^{42-44}$ Trend lines are included in both figures for clarity.

Error bars are drawn on the data points shown in Figs. 4 and 5 for both the penetration depths and the strains. The origin of the error in the penetration depth can be seen in Fig. 2 , where the error depends on the uncertainty in the incident angle and how close the incident angle is to the critical angle. Very near the critical angle the uncertainty in the penetration depth is greatest $(\sim 30 \mathrm{~nm})$. The uncertainty in the crystallographic strains arose from both systematic and random errors that occurred during the experiments. ${ }^{45-49}$ Great care was exercised to minimize systematic errors from instrument misalignment, specimen displacement, and beam divergence. Also, the random counting errors were minimized by taking the following three precautions:

(1) The experiments were operated in the count mode, and at least 100000 counts were collected from the incident beam for each data point;

(2) the data were collected in angular increments of $0.010^{\circ}-$ $0.015^{\circ}$; and 
(3) many of the diffraction peaks were collected multiple times.

Despite all of the precautions, uncertainty still exists, and the magnitude of the systematic error in the crystallographic strains was evaluated by a careful analysis of the $\mathrm{LaB}_{6}$ standard data. The variation in the strain $\partial \epsilon$ from an uncertainty in the peak position from random errors $\partial \theta$ was determined by differentiating Bragg's law. From a comparison of the magnitude of the systematic and random errors, the dominant factor was determined. The error bars in Figs. 3 and 4 were determined in this manner. For the sake of clarity, only one set of representative error bars for the crystallographic strains is shown in each plot. The errors in the strain eigenvalues, shown in Figs. 6 and 9, were determined using the approach described by Witte, Winholtz, and Neal, ${ }^{50}$ and from a more detailed analysis of the $\mathrm{LaB}_{6}$ standard data.

The strain eigenvectors resolved onto the laboratory reference frame as a function of penetration depth for the 50and 100-nm-thick film are shown in Fig. 6. The strains along the $x$ and $y$ directions in the laboratory frame, $\epsilon_{x}$ and $\epsilon_{y}$, remain relatively constant and compressive through the thickness of both films. This is in agreement with the inplane strain information obtained from the symmetric grazing incidence geometry. On the other hand, the normal component $\epsilon_{z}$ varies through each film thickness. The determination of the depth variation of the normal strain component is unique to this technique, and the analysis reveals that $\epsilon_{z}$ is very tensile near the free surface, and then becomes more compressive for larger penetration depths in both films. However, the differential between the azimuthal and normal strains near the free surface is greater for the 100 $\mathrm{nm}$ film. The strain may be more anisotropic near the free surface of the thicker film because the microstructure near the free surface becomes more anisotropic as the film thickness increases due to the development of grain facetting and a preferred growth direction. Specifically, previously published work showed that Mo films with a thickness of about $200 \mathrm{~nm}$ begin to develop a (110) out-of-plane growth texture, and films with a thickness of about $1 \mu \mathrm{m}$ begin to develop an in-plane alignment as well. ${ }^{37}$

It would be appropriate to compare the calculated value of $\epsilon_{z}$ corresponding to the full film thickness to the strain for a set of crystallographic planes parallel to the sample surface. This was accomplished with a $\theta-2 \theta$ scan of the $\{110\}$ and $\{220\}$ planes, where the scattering volume included the entire film thickness. The strain in both sets of planes was found to be $\sim 1.8 \times 10^{-3} \pm 5.0 \times 10^{-4}$, and the strain $\epsilon_{z}$ was $\sim 1.3 \times 10^{-3} \pm 6.0 \times 10^{-4}$, which displays good agreement in terms of sign and magnitude. Also, it is physically reasonable that the planes which are perpendicular and inclined to the sample surface are in compression, and the planes which are parallel to the surface are in tension.

A comparison of the average stresses obtained from this high-resolution diffraction technique is consistent with the average residual stress measurements with DCDT on these same films. The magnitude of the biaxial stress in the 50 and $100 \mathrm{~nm}$ Mo films obtained from $\mathrm{DCDT}^{33}$ and the average of $\sigma_{x}$ and $\sigma_{y}$, for the entire film thickness, determined with the
TABLE III. Comparison of average biaxial stresses through the entire film thickness determined by double-crystal diffraction topography (DCDT) and the high-resolution x-ray-diffraction method (HRXRD) presented in this article.

\begin{tabular}{ccc}
\hline \hline $\begin{array}{c}\text { Film } \\
\text { thickness }(\mathrm{nm})\end{array}$ & $\begin{array}{c}\text { DCDT stress } \\
(\mathrm{MPa})\end{array}$ & $\begin{array}{c}\text { HRXRD stress } \\
(\mathrm{MPa})\end{array}$ \\
\hline 50 & $-580 \pm 150$ & $-700 \pm 200$ \\
100 & $-250 \pm 100$ & $-280 \pm 150$ \\
\hline \hline
\end{tabular}

current technique is shown in Table III. The agreement is within the indicated error, but not exact, because the techniques are measuring different parameters. The DCDT technique measures the curvature of the $\mathrm{Si}$ substrate, from which the stress is calculated using a modified Stoney's equation. $^{11,12}$ For the Stoney's equation to be valid, there must be perfect continuity across the film/substrate interface. The present technique probes the strain in the Mo thin film directly, from which the stress was calculated. The determination of a larger average stress with this high-resolution technique would imply that the stress may be relaxing at the interface.

The average strains in a given slab throughout the 100nm-thick film are displayed as strain ellipsoids ${ }^{35}$ in Fig. 8, where the three-dimensional ellipsoids are shown as twodimensional cross sections. The dashed reference circles have a magnitude of unity and represent a volume element in the unstrained body. The solid ellipses represent the magnitude of the strains $\epsilon_{x}, \epsilon_{y}$, and $\epsilon_{z}$ in the laboratory reference frame in each slab. As usual, the radius of the ellipse in a direction that was subjected to a tensile strain is greater than unity, and the radius in a direction being subjected to a compressive strain is less than unity. The strains in the $x, y$, and $z$ directions were increased by 50 times so that the differential with the unit sphere is discernible. The actual strains determined for each slab are plotted in Fig. 9. This representation of the strains illustrates how much it can vary through the thickness of the film, which is not recognized when only an overall average value is determined.

One of the advantages of this technique is that once the strain eigenvalues and eigenvectors are determined for a particular frame of reference, they can be resolved onto any other reference frame of interest, because the strain state is completely defined. This technique also permits the determination of the depth dependence of the normal strain $\epsilon_{z}$, which cannot be accomplished with any particular scattering geometry. Finally, the strains can be related to other parameters, such as crystallographic directions in the film (if it is a single crystal or strongly textured), the rotation direction of the substrate during deposition, or deposition angles, to name a few. There are two limitations to this technique:

(1) The films must be crystalline; and

(2) a high-brightness X-ray source, such as a synchrotron, is needed to collect the six or more independent diffraction 
peaks required to solve the secular equation at shallow film penetration depths.

Such a source is also necessary to obtain a sufficient signalto-noise ratio and to enable the data to be collected in a reasonable amount of time.

\section{CONCLUSIONS}

This article describes a high-resolution synchrotron $\mathrm{x}$-ray-diffraction method to determine the complete strain tensor in a polycrystalline thin film, and uses the principle of total external reflection to profile the strains through the film thickness. The strain data are displayed physically in two different forms:

(1) as the data were collected, or strains as a function of penetration depth from the free surface of the film; and

(2) as strain ellipsoids present in successive slabs of the film.

In addition, the strains could be calculated for the growth mode, or as strains in a laboratory reference frame for volumes of material originating at the film/substrate interface. The present technique requires a detailed analysis of six or more independent $\{h k l\}$ diffraction peaks and was tested on two polycrystalline Mo films, with thicknesses of 50 and 100 $\mathrm{nm}$, which were deposited by planar magnetron sputtering onto $\mathrm{Si}$ (100) substrates. The results of this method were compared to DCDT, a macroscopic crystal lattice curvature technique, ${ }^{10}$ and the agreement was excellent.

In summary, the following was accomplished.

Absolute strain measurements were conducted on two Mo films, with thicknesses of 50 and $100 \mathrm{~nm}$, with a resolution in $\Delta d / d$ of $\sim 10^{-6}$.

The complete strain tensor was determined for the Mo films using a comprehensive high-resolution synchrotron $\mathrm{x}$-ray-diffraction technique. This procedure involved using the grazing incidence scattering geometry in the symmetric and asymmetric configurations.

The phenomenon of total external reflection was used to study the variation of the strain tensor in the Mo films as a function of $\mathrm{x}$-ray penetration depth, by varying the incident angle of the $\mathrm{x}$ rays near the critical angle for total external reflection.

The data were displayed as strains (and stresses) in a laboratory reference frame as a function of penetration depth from the free surface of the film. A linear elastic model was used to convert the strain data, as a function of depth, into the strain ellipsoids present in successive slabs of the film.

The values of the average strains in the laboratory reference frame, through the entire thickness of the $100 \mathrm{~nm}$ film, were $\epsilon_{x}=-0.00085, \epsilon_{y}=-0.00085$, and $\epsilon_{z}=0.00132$, respectively. The strains for the top $5 \mathrm{~nm}$ of the same film were $\epsilon_{x}=-0.00019, \epsilon_{y}=-0.00301$, and $\epsilon_{z}=0.00460$, respectively.

From the above observations, a large anisotropy between the in-plane and out-of-plane strains is apparent, especially near the free surface. It is also apparent that a large gradient in $\epsilon_{z}$ exists near the free surface of the $100 \mathrm{~nm}$ film.
The average stress for the full Mo film thickness was in excellent agreement with that measured on the same samples via DCDT.

\section{ACKNOWLEDGMENTS}

This work was supported by the USARO and ARPA under Contract No. DAAL03-91-0235 and DAAH04-95-10120. The synchrotron work was conducted at the Stanford Synchrotron Radiation Laboratory, funded by the U.S. DOE. The RBS work was conducted at the Michigan Ion Beam Laboratory, and the DCDT experiments were conducted by J. G. Hershberger and L. Parfitt. The authors wish to thank J. Kulman at the University of Michigan Solid State Electronics Laboratory and S. Brennan at SSRL. One of us (S.G.M.) would like to thank Dr. A. K. Malhotra and Dr. R. A. Winholtz for helpful discussions.

${ }^{1}$ J. A. Floro, C. V. Thompson, R. Carel, and P. D. Bristowe, J. Mater. Res. 9, 2411 (1994).

${ }^{2}$ H. Windischmann, Crit. Rev. Solid State Mater. Sci. 17, 547 (1992).

${ }^{3}$ W. D. Nix, Metall. Trans. A 20, 2217 (1989).

${ }^{4}$ D. J. Morrison, J. W. Jones, G. S. Was, A. Mashayekhi, and D. W. Hoffman, in Thin Films: Stresses and Mechanical Properties, edited by John C. Bravman, William D. Nix, David M. Barnett, and David. A. Smith, Mater. Res. Symp. Proc., Vol. 130 (MRS, Pittsburgh, PA, 1989), p. 53.

${ }^{5}$ P. A. Flinn, Mater. Res. Symp. Proc. 130, 41 (1989).

${ }^{6}$ M. Renninger, Phys. Lett. 1, 104 (1962).

${ }^{7}$ M. Renninger, Z. Phys. 19, 20 (1965).

${ }^{8}$ M. Renninger, Z. Naturforsch. 160, 1110 (1961).

${ }^{9}$ C. L. Kuo, P. E. Vanier, and J. C. Bilello, J. Appl. Phys. 55, 375 (1984).

${ }^{10}$ J. Tao, L. H. Lee, and J. C. Bilello, J. Electron. Mater. 20, 819 (1991).

${ }^{11}$ G. G. Stoney, Proc. R. Soc. London Ser. A 82, 172 (1909).

${ }^{12}$ D. W. Hoffman, Phys. Thin Films 3, 211 (1965).

${ }^{13}$ C. S. Barrett and T. B. Massalski, Structure of Metals, 3rd ed. (McGrawHill, New York, 1966), pp. 466-485.

${ }^{14}$ H. P. Klug and L. E. Alexander, X-Ray Diffraction Procedures (Wiley, New York, 1974), pp. 755-790.

${ }^{15}$ L. H. Schwartz and J. B. Cohen, Diffraction from Materials, 2nd ed. (Springer, New York, 1987), pp. 372-381.

${ }^{16}$ M. R. James and J. B. Cohen, Treatise on Materials Science and Technology, edited by Herbert Herman (Academic, New York, 1980), Vol. 19A, pp. 2-62.

${ }^{17}$ I. C. Noyan and J. B. Cohen, in Residual Stress and Stress Relaxation, edited by Eric Kula and Volker Weiss, Sagamore Army Materials Research Conference Proceedings (Plenum, New York, 1982), pp. 1-17.

${ }^{18}$ H. Dölle and V. Hauk, Z. Metal. 68, 728 (1977).

${ }^{19}$ I. C. Noyan and J. B. Cohen, Residual Stress Measurement by Diffraction and Interpretation (Springer, New York, 1987), pp. 117-163.

${ }^{20}$ W. C. Marra, P. Eisenberger, and A. Y. Cho, J. Appl. Phys. 50, 6927 (1979).

${ }^{21}$ P. Eisenberger and W. C. Marra, Phys. Rev. Lett. 46, 1081 (1981).

${ }^{22}$ P. H. Fouss and S. Brennan, Ann. Rev. Mater. Sci. 20, 365 (1990).

${ }^{23}$ L. J. Martinez-Miranda, J. J. Santiago-Aviles, W. R. Graham, P. A. Heiney, and M. P. Siegal, J. Mater. Res. 9, 1434 (1994).

${ }^{24}$ R. Venkatraman, P. R. Besser, J. C. Bravman, and S. Brennan, J. Mater. Res. 9, 328 (1994).

${ }^{25}$ P. R. Besser, S. Brennan, and J. C. Bravman, J. Mater. Res. 9, 13 (1994).

${ }^{26}$ C. J. Shute and J. B. Cohen, J. Appl. Phys. 70, 2104 (1991).

${ }^{27}$ T. Imura, S. Weissmann, and J. J. Slade, Jr., Acta. Crystallogr. 15, 786 (1962)

${ }^{28}$ J. A. Bain, L. J. Chyung, S. Brennan, and B. M. Clemens, Phys. Rev. B 44, 1184 (1991)

${ }^{29} \mathrm{Ph}$. Goudeau, K. F. Badawi, A. Naudon, and G. Gladyszewski, Appl. Phys. Lett. 62, 246 (1993).

${ }^{30}$ L. Maniguet, M. Ignat, M. Dupeux, P. A. Flinn, Ph. Normandon, P. Gergaud, and J. J. Bacmann, Advanced Metallization for ULSI Applications, edited by T. S. Cale and F. S. Pintchovski, Mater. Res. Soc. Symp. Proc., Vol. VIII (MRS, Pittsburgh, PA, 1992), p. 67. 
${ }^{31}$ M. Vill, D. P. Adams, S. M. Yalisove, and J. C. Bilello, Acta. Metall. mater. 43, 427 (1995).

${ }^{32}$ C. Montcalm, B. T. Sullivan, H. Pepin, J. A. Dobrowolski, and M. Sutton, Appl. Opt. 33, 2057 (1994).

${ }^{33}$ L. J. Parfitt (private communication).

${ }^{34}$ H. Dosch, Phys. Rev. B 35, 2137 (1987).

${ }^{35}$ J. F. Nye, Physical Properties of Crystals (Oxford University Press, Oxford, 1985), pp. 41-43.

${ }^{36}$ G. E. Deiter, Mechanical Metallurgy (McGraw-Hill, New York, 1961), p. 39.

${ }^{37}$ O. P. Karpenko, J. C. Bilello, and S. M. Yalisove, J. Appl. Phys. 76, 4610 (1994).

${ }^{38}$ D. P. Adams, L. J. Parfitt, J. C. Bilello, S. M. Yalisove, and Z. U. Rek, Thin Solid Films (to be published).

${ }^{39}$ H. Dölle, J. Appl. Crystallogr. 12, 489 (1979).

${ }^{40}$ I. C. Noyan, Metall. Trans. A 14, 249 (1983).

${ }^{41}$ M. F. Doerner and S. Brennan, J. Appl. Phys. 63, 126 (1988).

${ }^{42}$ R. M. Fisher, J. Z. Duan, and A. G. Fox, in Thin Films: Stresses and
Mechanical Properties, edited by John C. Bravman, William D. Nix, David M. Barnett, and David A. Smith, Mater. Res. Soc. Symp. Proc., Vol. 130 (MRS, Pittsburgh, PA, 1989), p. 249.

${ }^{43}$ R. W. Hoffman, Surf. Interface Anal. 3, 62 (1981)

${ }^{44}$ T. J. Vink, M. A. J. Somers, J. L. C. Daams, and A. G. Dirks, J. Appl. Phys. 70, 4301 (1991).

${ }^{45}$ R. A. Winholtz and J. B. Cohen, Aust. J. Phys. 41, 189 (1988).

${ }^{46}$ M. R. James and J. B. Cohen, Advances in X-Ray Analysis (Plenum, New York, 1776), Vol. 20, pp. 291-305.

${ }^{47}$ C. J. Kelly and M. A. Short, Advances in X-Ray Analysis (Plenum, New York, 1970), Vol. 14, pp. 377-388.

${ }^{48}$ C. F. Jatczak and H. F. Boehm, Advances in X-Ray Analysis (Plenum, New York, 1973), Vol. 17, pp. 354-370.

${ }^{49}$ H. Zantopulos and C. F. Jatczak, Advances in X-Ray Analysis (Plenum, New York, 1970), Vol. 14, pp. 360-376.

${ }^{50}$ D. A. Witte, R. A. Winholtz, and S. P. Neal, Advances in X-Ray Analysis (Plenum, New York, 1994), Vol. 37, pp. 265-277. 